\title{
Multi-Constraint QoS Routing Using a New Single Mixed Metric
}

\author{
Pejman Khadivi ${ }^{1,2}$ \\ Shadrokh Samavi ${ }^{1,2}$ \\ ${ }^{1}$ Department of Electrical and Computer Engineering \\ McMaster University \\ Hamilton, Ontario, Canada L8S 4K1 \\ \{samavi, todd\}@mcmaster.ca
}

\author{
Terence D. Todd ${ }^{1}$ \\ Hossein Saidi ${ }^{2}$ \\ 2 Department of Electrical and Computer Engineering \\ Isfahan University of Technology \\ Isfahan, IRAN \\ hsaidi@cc.iut.ac.ir
}

\begin{abstract}
Multi-constraint quality-of-service routing will become increasingly important as the Internet evolves to support real-time services. It is well known however, that optimum multi-constraint QoS routing is computationally complex, and for this reason various heuristics have been proposed for routing in practical situations. Among these methods, those that use a single mixed metric are the most popular. Although mixed metric routing discards potentially useful information, this is compensated for by significantly reduced complexity. Exploiting this tradeoff is becoming increasingly important where low complexity designs are desired, such as in battery operated wireless applications. In this paper, a novel single mixed metric multi-constraint routing algorithm is introduced. The proposed technique has similar complexity compared with existing low complexity methods. Simulation results are presented which show that it can obtain better performance than comparable techniques in terms of generating feasible multi-constraint QoS routes.
\end{abstract}

\section{INTRODUCTION}

Routing is one of the most basic and widely studied problems in computer networking. The current Internet however, uses only best-effort routing [1] and thus supports services without any quality of service (QoS) guarantees. Applications such as real-time audio and video however, require strict performance guarantees in order to achieve acceptable performance. For these types of applications, a fundamental issue is how to find a feasible path that satisfies multiple constraints. This problem is known as multiconstraint QoS routing.

QoS routing is very complex, and dealing with multiple QoS requirements makes this problem NP-Complete [2]. In the multi-constraint case, each network link has multiple weights which can be classified as additive, multiplicative or concave. For additive weights, the end-to-end weight of the path is the sum of the individual link values. Delay is an example of additive weights. A multiplicative path weight is the product of the link values along the path. Path reliability is an example of multiplicative weights. Bandwidth belongs to the class of concave weights. The overall bandwidth of the path is equal to the minimum bandwidth of the links. Dealing with concave weights and constraints is very easy. It can be proven that optimum QoS routing with more than one constraint involving additive and/or multiplicative weights is an NP-Complete problem. For this reason it is difficult to have an algorithm which is computationally efficient in all possible situations [2]. In this paper we use weight and metric as synonymous. Since it is possible to transform the multiplicative weight case into the additive case by taking logarithms, we only consider cases with several additive constraints. Hence, the Multi-Constraint Path (MCP) problem can be stated as follows.

Definition: Consider a network that is represented by a directed or undirected graph $G=(V, E)$, where $V$ is the set of nodes and $E$ is the set of links. Each link $(i, j) \in E$ has $K$ additive non-negative QoS weights, $w_{m}(i, j), m=1, \ldots, K$. Given $K$ constraints, $C_{m}, m=1, \ldots, K$, the MCP problem is to find a path $\mathrm{P}$ from a source node $\mathrm{S}$ to a destination node $\mathrm{D}$ such that,

$$
w_{m}(P) \hat{=} \sum_{(i, j)} w_{m}(i, j) \leq C_{m}
$$

There are various methods for solving the MCP problem. Depth-First Search (DFS) is one approach. Although this method is able to find a feasible path if one exists, its worsecase time complexity is exponential. Shin and his co-workers have suggested a heuristic based on DFS which limits the number of crankbacks to control the worst case time complexity. Even though the time complexity is reduced it is possible that the algorithm will not find a feasible path even when one exists [3].

There are a number of methods that use a single mixed metric instead of dealing with multiple link weights. According to Wang and Crowcroft [2], a single mixed metric method at best can be used as criterion in path selection but it does not contain sufficient information alone to determine if QoS requirements are satisfied. However, using a mixed metric can reduce the time complexity because we can employ a single source single destination shortest path algorithm such as Dijkstra's algorithm, but this is not as effective as it may appear. When a single mixed metric is used for routing, some information is lost [4][5][6][7]. The TAMCRA algorithm presented in [5] uses a single metric 
and a $k$-shortest path algorithm in order to solve a MCP problem. The k-shortest path algorithms are able to find multiple shortest paths between a given source and a destination. This method reduces the performance shortcomings of using a mixed metric. Other routing algorithms also exist for solving the MCP problem, based on mixed metrics, manipulated weights or distributed algorithms and flooding [8][9][10][11][12][13][14][15].

In this paper, we propose a novel algorithm for the MCP problem using a single mixed metric. Simulation results show that our proposed method can have better performance than existing algorithms with a similar computational complexity. The remainder of the paper is organized as follows. In Section 2 a brief review is presented of MCP routing based on a single mixed metric. In Section 3 our new algorithm is proposed. Simulation results are presented in Section 4 and some concluding remarks are given in Section 5 .

\section{Routing Based on A Single MiXed Metric}

In [4] the following mixed metric was introduced for the two-constraint problem,

$$
w(e)=d_{1} w_{1}(e)+d_{2} w_{2}(e)
$$

where $e$ is a link with two different metrics, $w_{1}(e)$ and $w_{2}(e)$. Here, $d_{1}$ and $d_{2}$ are two constants and $w(e)$ is the mixed metric associated with link $e$. This formulation is known as Jaffe's method. If a graph has weights given by $w(e)$, and a shortest path algorithm such as Dijkstra's Algorithm is used, then for a path $p=(S, u, \ldots, v, D)$, the path weight $w(p)$ can be written as follows,

$$
\begin{aligned}
& w(p)=\left[d_{1} w_{1}(S, u) \dashv d_{2} w_{2}(S, u)\right] \dashv \ldots \dashv\left[d_{1} w_{1}(v, D)\right. \\
& \left.+d_{2} w_{2}(v, D)\right]=d_{1}\left[w_{1}(S, u)+\ldots+w_{1}(v, D)\right] \\
& +d_{2}\left[w_{2}(S, u)+\ldots w_{2}(v, D)\right]
\end{aligned}
$$

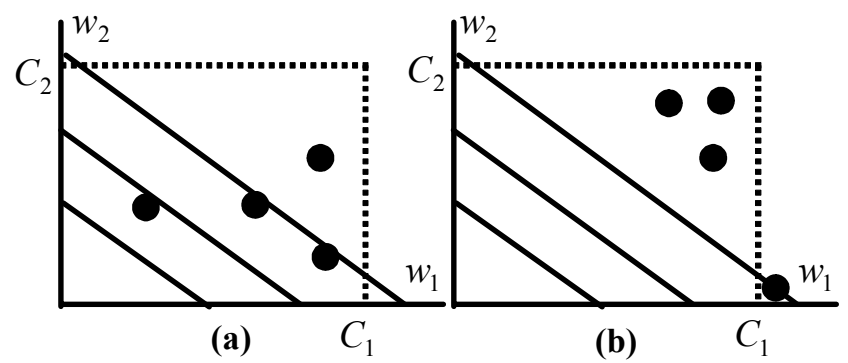

Figure 1. Use of a single mixed metric. (a) feasible solution is found (b) algorithm fails.

where $w(p)$ is minimal. Equation (3) describes parallel lines such as, $d_{1} w_{1}(p) \dashv d_{2} w_{2}(p)=c$. These parallel lines are illustrated in Fig. 1 and show how this method searches for a feasible path. In this figure the horizontal axis is associated with the $w_{1}$ metric and the vertical axis is associated with $w_{2}$. The objective is to find a path, $p^{*}$, such that $w_{1}\left(p^{*}\right) \leq C_{1}$ and $w_{2}\left(p^{*}\right) \leq C_{2}$. Constraints $C_{1}$ and $C_{2}$ are shown in Fig. 1 as dashed lines. Each path $p$ between source $S$ and destination $D$ has weights $w_{1}(p)$ and $w_{2}(p)$. Therefore, associated with each path is an achievable point in the $w_{1}(p)-w_{2}(p)$ plane. In Fig. 1 there are some examples of these paths shown as black points. Clearly all of the points inside the rectangular region are associated with feasible paths and the Jaffe method searches for a path with the minimum $w(p)$. Fig. 1(a) illustrates a situation where the Jaffe method finds a feasible path, i.e., the point shown closest to the origin. Since using mixed metrics discards some useful information, it is possible for the Jaffe method to fail. Fig. 1(b), shows a simple case where this happens, i.e., the right-most path found does not satisfy the constraints.

An approach for improving the chance of finding a feasible path is to modify the path weight in order to influence the search region. For example, if $d_{1}$ and $d_{2}$ are selected based on $d_{1} / d_{2}=C_{2} / C_{1}$ then before leaving the feasible path region, half of it will be searched [5]. It is also possible to define the following weight for a path,

$$
W_{\lambda}(p)=\sum_{j=1}^{K}\left(w_{j} / C_{j}\right)^{\lambda}
$$

where $p$ is a path that minimizes the mixed metric $W_{\lambda}$ for a given $\lambda \geq 1$. Fig. 2 illustrates how the feasible path search region is affected by an algorithm that uses (4), with two constraints and $\lambda=2$ to find its mixed weights. It is clear from this figure that when we use squaring in the mixed weight computation, it will take longer for the algorithm to leave the feasible path region. It was proven in [6] that after using a mixed metric algorithm for a MCP problem in order to find a path $p$, when a feasible path $p^{*}$ exists then,

$$
\begin{cases}W_{j}(p) \leq C_{j} \quad \text { for } \quad \text { some } j \\ W_{j}(p) \leq \sqrt[\lambda]{K} C_{j} \quad \text { for others }\end{cases}
$$

It is clear that larger values of $\lambda$ lead to a higher probability of obtaining feasible results. It has also been proven that when $\lambda-\propto$ the following metric can be used [6],

$$
W_{\infty}(p)=\max \left\{w_{1}(p) / C_{1}, \ldots, w_{K}(p) / C_{K}\right\}
$$

\section{PROPOSED METHOD}

We first consider the mixed metric routing method when there are only two constraints and $\lambda=1$. As shown in Fig. 3 we assume a situation where paths $t$ and $q$ are between the 
source and destination nodes. Path $t$ is a feasible path since it satisfies the constraints. On the other hand, path $q$ minimizes the mixed metric but it does not satisfy the constraints. The mixed metric of path $t$ is $W(t)=w_{1}(t) / C_{1} \dashv w_{2}(t) / C_{2}$ and for the path $q, W(q)=w_{1}(q) / C_{1} \dashv w_{2}(q) / C_{2}$ and also we have $W(q)<W(t)$ while path $t$ satisfies both constraints. Based on (5), path $q$ at least satisfies one of the constraints. Assume $w_{1}(q) / C_{1} \leq 1$. Then we can write:

$$
\frac{w_{1}(q)}{C_{1}}<\frac{w_{1}(t)}{C_{1}}+\frac{w_{2}(t)}{C_{2}}-\frac{w_{2}(q)}{C_{2}}
$$

but since $\left(w_{2}(t) / C_{2}\right)<\left(w_{2}(q) / C_{2}\right)$, based on (7):

$$
\frac{w_{1}(q)}{C_{1}}<\frac{w_{1}(t)}{C_{1}}
$$

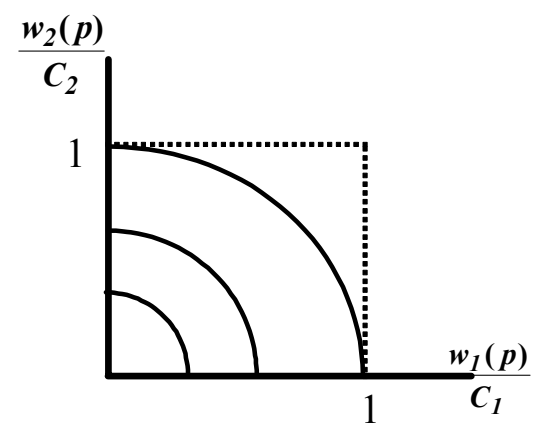

Figure 2. Use of a quadratic single mixed metric.

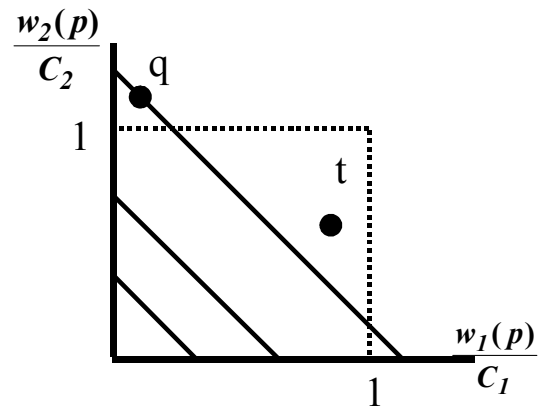

Figure 3. A feasible path exists but is not found.

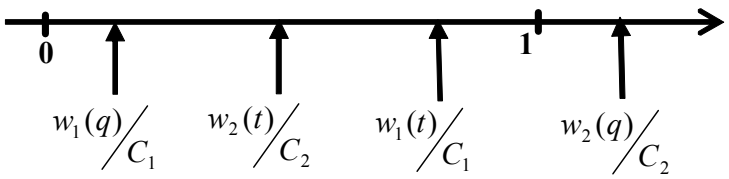

Figure 4. Relative positions of the parameters in (14) and (15).

Fig. 4 shows the relative position of the above four ratios. In the general multi-constraint case we define:

$$
\mu(p)=\frac{1}{K} \sum_{i=1}^{K} w_{i}(p) / C_{i}
$$

and

$$
\Delta(p)=\sum_{i=1}^{K}\left(\frac{w_{i}(p)}{C_{i}}-\mu(p)\right)^{2}
$$

It is clear that for the example of Fig. 3 with two constraints that $\mu(t)>\mu(q)$ and $\Delta(t)<\Delta(q)$.

In existing algorithms, routing for a path, $p$, is based only on $\mu(p)$. We propose to use $\Delta(p)$ as well as $\mu(p)$ in the routing decisions. Hence, we define the following mixed metric,

$$
G(p) \hat{=} \mu(p)[\Delta(p) \dashv \varepsilon]
$$

where $\varepsilon$ is a constant and $0 \leq \varepsilon \leq 1$. By using $G(p)$ as a single metric, both $\mu(p)$ and $\Delta(p)$ are considered. A constant coefficient such as $\varepsilon$ allows $\mu(p)$ to have a direct effect on the solution found. In addition, the product $\mu(p) \Delta(p)$ is taken into account and obviously larger values of $\mu(p)$ and $\Delta(p)$ increases $G(p)$. The constant $\mathcal{E}$ achieves the proper weighting for the role of $\mu(p)$. The best value of $\varepsilon$ is dependent upon network size and its weights.

A complete description of the routing algorithm is shown in Fig. 5. The algorithm operates as a modified version of Dijkstra's shortest path algorithm, however in this case $G(p)$ is used only as an indicator function. An alternative is to apply (11) for each link, $e$, i.e., and then use $G(e)$ as the link weight for $e$. Simulation results show that using $G(p)$ is advantageous over $G(e)$.

In the algorithm description shown in Fig. 5, SRC and DEST are the source and destination nodes. In each node $\mathrm{V}$ of the network, $\mathrm{W}_{\mathrm{j}}[\mathrm{V}]$ is the $j$-th weight of the selected path between SRC and V. Also, $\mu[\mathrm{V}]$ is the average of $\mathrm{W}_{\mathrm{j}}[\mathrm{V}]$ for all $j$ 's. The corresponding $\Delta$ function is $\Delta(\mathrm{V})$. We define PREVIOUS[V] to hold the previous node of $\mathrm{V}$ when traversing on the path between SRC and $\mathrm{V}$. The parameter $\mathrm{L}(\mathrm{V}, \mathrm{B})$ gives the length between nodes $\mathrm{V}$ and $\mathrm{B}$. PERMANENT[V] indicates if $\mathrm{V}$ is a permanent node. TAG is the node whose neighbors are currently being examined. This description is similar to that commonly used for Dijkstra's algorithm.

It can be shown that the complexity of the overall algorithm is $O\left(K N^{2}\right)$ where $\mathrm{N}$ is the number of network nodes. The complexity of the single mixed metric algorithms with $\lambda>1$ is not better because their structure does not differ from the new one and the difference is in the mixing functions. Hence, our new algorithm is comparable with 
existing low complexity algorithms in terms of time complexity.

\section{Simulation ResUlts}

A large variety of simulation experiments have been performed using a wide range of different parameter values. In this section some representative results are presented which illustrate the relative performance of the proposed algorithm. In the results to be presented, the performance measure used for comparison is the Success Ratio (SR), which is defined as the percentage of time that the algorithm finds a feasible path when at least one exists. Single mixed metric routing methods introduced in Section 2, for $1 \leq \lambda \leq 4$ are simulated as well as our proposed algorithm. In addition, the method based on (6) is simulated. In all of the existing algorithms of Section 2, the single mixed metric is used only as a path indicator.

Random network topologies for the simulations were generated using Waxman's method [16]. After generating each topology, weights for each link were selected randomly. For a link $e, w_{1}(e)$ is a uniformly selected random number from $[0,5]$ or from $[0,50]$, and $w_{2}(e)$ is uniformly selected from $[0,10]$ or $[0,200]$. Source, destination and constraints are generated based on the method of [6]. The source and destination are randomly generated such that the minimum hop-count between them is at least three. If $p$ and $q$ are the two shortest paths between the source and destination, and using weights $w_{1}$ and $w_{2}$, then the constraint $C_{1}$ is uniformly selected from $\left[0.8 w_{1}(q), 1.2 w_{1}(q)\right]$ and $C_{2}$ is uniformly selected from $\left[0.8 w_{2}(p), 1.2 w_{2}(p)\right]$. Networks with $10,15,20,25,30,35$ and 40 nodes are used and simulation results are shown for the 2-constraint cases.

Fig. 6 indicate that the proposed algorithm achieves better performance than the other mixed metric methods with $\lambda=4$ and is comparable with the algorithm that is based on (6). Fig. 7 shows the importance of the parameter, $\varepsilon$. When $\varepsilon$ is zero, the performance of our method is very poor compared with the linear combination of weights. Also, the effect of $\varepsilon$ is shown in Figs. 8 and 9 where the value of the single mixed metric function $(G)$ is shown for a twoconstraint case. It is clear from these figures that when $\varepsilon=0.5$, the value of the single metric function inside the feasible solution area is always less than its value outside of this area. This is not true when $\varepsilon=0$.

Fig. 7, also shows the case where the new mixed metric is not used as a path indicator but is used for computing a single weight for each link. This situation is compared with the one that deploys a mixed metric only as a path indicator. When the mixed metric function that is a non-linear function of the weights is used as a path indicator, better performance is obtained.

ROUTING ALGORITHM

\section{(NETWORK, SRC, DEST, CONSTRAINTS}

\{SRC is the source node. DEST is the destination node\}

$\left\{L_{j}(U, V)\right.$ is the j-th weight of the link between nodes $U$ and $\left.V\right\}$

BEGIN

01 FOR (all nodes $V$ ) DO

02 BEGIN

$04 \quad \mathrm{~W}_{\mathrm{j}}[V] \leftarrow \infty$;

$05 \mu(V) \leftarrow \infty$;

$06 \Delta(V) \leftarrow \infty$;

$07 \quad$ PREVIOUS $(V) \leftarrow \mathrm{NULL}$;

$08 \quad$ PERMANENT $(V) \leftarrow$ FALSE;

09 END;

10 FOR ( $\mathrm{j}=1$ to $\mathrm{K})$ DO

$11 \quad W_{j}[S R C] \leftarrow 0$;

$12 \mu[S R C] \leftarrow 0$;

13 PREVIOUS $[S R C] \leftarrow$ NULL;

14 PERMANENT $[S R C] \leftarrow$ TRUE;

$15 T A G \leftarrow S R C$;

16 WHILE $(T A G \neq D E S T)$ DO

17 BEGIN

18 FOR (all nodes, $V$, neighbors of $T A G$ ) DO

19 BEGIN

20

21

22

23

24

25

26

27

28

29

30

31

32

33

34

35

36

37

38

39 END;

END.

Figure 5. Proposed Multi-Constraint routing algorithm

\section{CONCLUSIONS}

In this paper a new single mixed metric routing algorithm was presented for solving the MCP problem. The proposed method takes into account variations of the link weights in performing path selection, and the results suggest that this is as important as the linear combination of these weights. The 
proposed algorithm uses a parameter, $\varepsilon$, that helps control the region over which searching is performed. It is possible to use the single mixed metric function as either a path indicator or for computing a single weight for each link. Better results may be obtained if the single mixed is used as a path indicator.

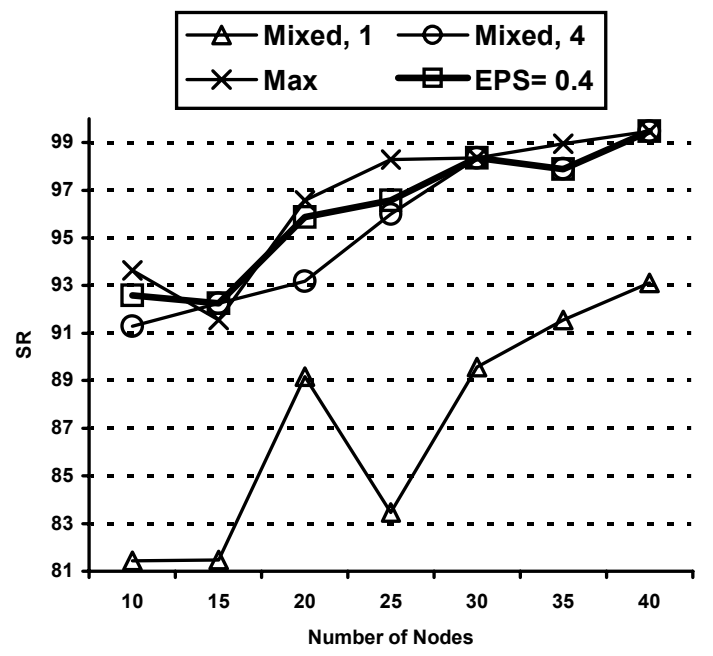

Figure 6. SR with fixed $\varepsilon$
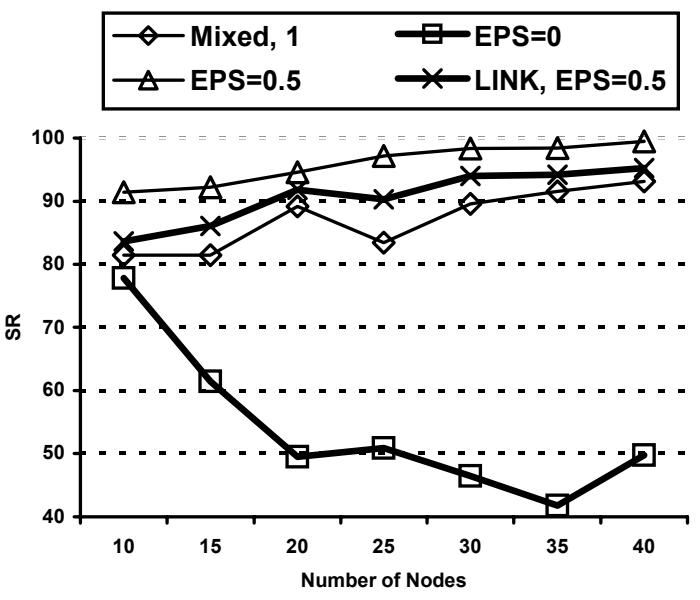

Figure 7. The effect of $\mathcal{\varepsilon}$

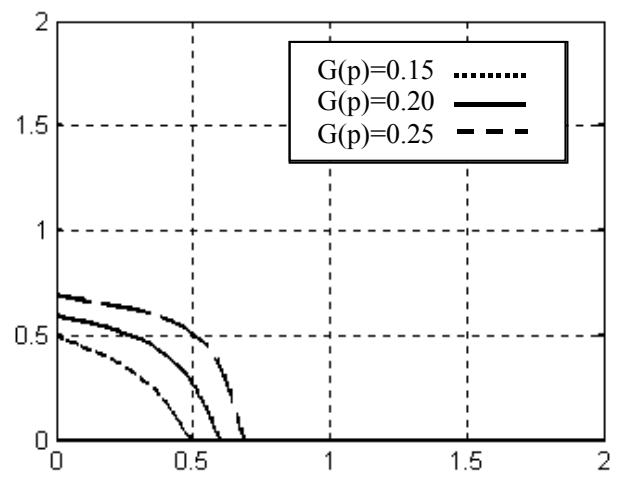

Figure 8. Single mixed metric function in a two constraint case for $\varepsilon=0.5$

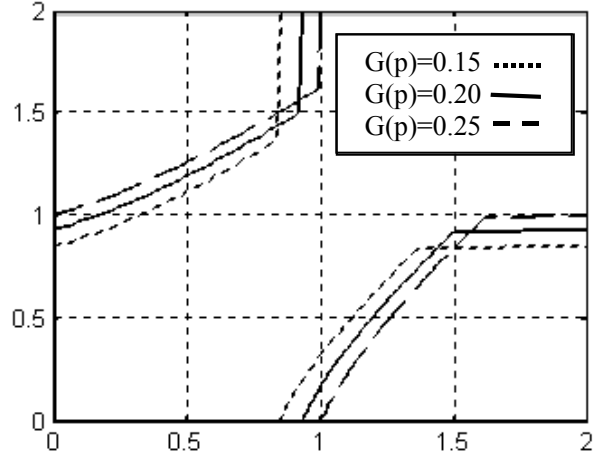

Figure 9. Single mixed metric in a two constraint case for $\varepsilon=0$

\section{REFERENCES}

[1] Z. Wang, Internet QoS: Architectures and Mechanisms for Quality of Service, Morgan Kaufmann Publishers, 2001

[2] Z. Wang, J. Crowcroft "Quality-of-service routing for supporting multimedia applications", IEEE Journal on Selected Areas in Communications, Vol. 14, Pp. 1228 -1234, Sept. 1996

[3] D.W. Shin, E.K.P.Chong, H.J.Siegel, “A multiconstraint QoS routing scheme using the depth-first search method with limited crankbacks", IEEE Workshop on High Performance Switching and Routing, Pp. $385-389,2001$

[4] J.M. Jaffe, "Algorithms for Finding Paths with Multiple Constraints", Networks, Vol. 14, Pp. 95-116, 1984

[5] H. Neve, P. Mieghem, "A multiple quality of service routing algorithm for PNNI", 1998 IEEE ATM Workshop Proceedings, Pp. $324-328,1998$

[6] T. Korkmaz, M. Krunz "Multi-constrained optimal path selection", Proceedings of INFOCOM 2001, Vol.2, Pp. 834 -843, 2001

[7] L.Costa, S.Fdida, O.Duarte, "A scalable algorithm for link-state QoSbased routing with three metrics", IEEE International Conference on Communications ICC 2001, Vol.8, Pp. 2603 -2607, 2001

[8] G. Feng, C.Douligeris, K. Makki, N. Pissinou, "Performance evaluation of delay-constrained least-cost QoS routing algorithms based on linear and nonlinear lagrange relaxation", IEEE International Conference on Communications ICC 2002, Vol.4, Pp. $2273-2278,2002$

[9] A. Juttner, B. Szviatovski, I. Mecs, Z. Rajko, "Lagrange relaxation based method for the QoS routing problem", Proceedings INFOCOM 2001, Vol. 2, Pp. 859-868, 2001

[10] G. Feng, K. Makki, N. Pissinou, C. Douligeris, "An efficient approximate algorithm for delay-cost-constrained QoS routing", Proceedings of the Tenth International Conference on Computer Communications and Networks, Pp. $395-400,2001$

[11] L. Guo, I. Matta, "Search space reduction in QoS routing", Proceedings of the 19th IEEE International Conference on Distributed Computing Systems, Pp. $142-149,1999$

[12] X. Yuan, X. Liu, "Heuristic algorithms for multi-constrained quality of service routing", Proceedings of INFOCOM 2001, Vol. 2, Pp. 844 $-853,2001$

[13] S. Chen, K. Nahrstedt, "On finding multi-constrained paths", IEEE International Conference on Communications ICC 98, Vol. 2, Pp. 874 $-879,1998$

[14] J. Song, H. Pung, L. Jacob, "A multi-constrained distributed QoS routing algorithm", IEEE International Conference on Networks ICON 2000, Pp. $165-171,2000$

[15] A. Fei, M. Gerla, "Smart forwarding technique for routing with multiple QoS constraints", IEEE Global Telecommunications Conference GLOBECOM '00, Vol. 1, Pp. 599 -604, 2000

[16] B.M. Waxman, "Routing of Multipoint Connections", IEEE Journal on Selected Areas in Communications, Vol. 6, No. 9, Pp. 1617-1622, 1988 